\title{
Statistics of the detection rates for tensor and scalar gravitational waves from the Local Galaxy universe
}

\author{
Yu. V. Baryshev ${ }^{1,2}$ and G. Paturel ${ }^{3}$ \\ 1 Astronomical Institute of the Saint-Petersburg University, 198504 St.-Petersburg, Russia \\ 2 Isaac Newton Institute of Chile, Saint-Petersburg Branch, Russia \\ 3 CRAL-Observatoire de Lyon, 69561 Saint-Genis Laval Cedex, France
}

Received 19 May 2000 / Accepted 7 March 2001

\begin{abstract}
We use data on the local 3-dimensional galaxy distribution for studying the statistics of the detection rates of gravitational waves (GW) coming from supernova explosions. We consider both tensor and scalar gravitational waves which are possible in a wide range of relativistic and quantum gravity theories. We show that statistics of GW events as a function of sidereal time can be used for distinction between scalar and tensor gravitational waves because of the anisotropy of spatial galaxy distribution. For calculation of the expected amplitudes of GW signals we use the values of the released GW energy, frequency and duration of GW pulse which are consistent with existing scenarios of SN core collapse. The amplitudes of the signals produced by Virgo and the Great Attractor clusters of galaxies is expressed as a function of the sidereal time for resonant bar detectors operating now (IGEC) and for forthcoming laser interferometric detectors (VIRGO). Then, we calculate the expected number of GW events as a function of sidereal time produced by all the galaxies within $100 \mathrm{Mpc}$. In the case of axisymmetric rotational core collapse which radiates a GW energy of $10^{-9} M_{\odot} c^{2}$, only the closest explosions can be detected. However, in the case of nonaxisymmetric supernova explosion, due to such phenomena as centrifugal hangup, bar and lump formation, the GW radiation could be as strong as that from a coalescing neutron-star binary. For radiated GW energy higher than $10^{-6} M_{\odot} c^{2}$ and sensitivity of detectors at the level $h \approx 10^{-23}$ it is possible to detect Virgo cluster and Great Attractor, and hence to use the statistics of GW events for testing gravity theories.
\end{abstract}

Key words. gravitation - relativity - waves - supernovae: general - galaxies: - clusters: general

\section{Introduction}

In a few years the third generation of gravitational wave detectors will start searching for the most energetic events in the Universe caused by gravitational collapse and merging of relativistic compact massive objects (see the review by Thorne 1997). This opens a new window onto the Universe and creates new connections between optical extragalactic astronomy and gravitational wave astronomy. This will be the beginning of genuine observational study of the physics of the core collapse supernova explosions and testing relativistic and even quantum gravity theories (Damour 1999; Gasperini 1999).

Expected sources of powerful gravitational wave (hereafter, GW) events are connected with supernova explosions and merging of neutron stars and other relativistic compact massive objects in galaxies. Predicted GW signals essentially depend on the details of the last relativistic stages of the gravitational collapse which is still poorly

Send offprint requests to: G. Paturel, e-mail: patu@obs.univ-lyon1.fr known (Thorne 1987, 1997; Paczyncki 1999; Burrows 2000). Moreover studies of scalar-tensor gravity theories have shown that spherical gravitational collapse and binary systems generate scalar GWs which may be detected by existing GW detectors (Baryshev 1982, 1995, 1997; Baryshev \& Sokolov 1984; Sokolov 1992; Damour \& Esposito-Fareze 1992, 1996, 1998; Shibata et al. 1994; Harada et al. 1997; Bianchi et al. 1998; Damour 1999; Brunetti et al. 1999; Gasperini 1999; Novak \& Ibanez 1999; Maggiore \& Nicolis 2000; Nakao et al. 2000).

The aim of this paper is to estimate the contribution of nearby galaxies and clusters of galaxies, within a radius of about $100 h_{60}^{-1} \mathrm{Mpc}$ around our Galaxy, to the detection of the possible GW events. This requires the knowledge of the actual 3-dimensional galaxy distribution (Sect. 2), the intrinsic rate of the most powerful events expected from different galaxy types (Sect. 3), and the amplitude of the GW signal according to the prediction of existing scenarios of SN core collapses (Sect. 4). In Sect. 5 we calculate the probability of GW events as a function of sidereal time for currently operating bar detectors and forthcoming 
interferometric detectors. In this section we first study the amplitude expected for the Virgo cluster and the Great Attractor and then derive the density of probability of GW events as a function of the sidereal time for some detectors. A discussion of the results and the main conclusions are given in Sect. 6.

\section{Distribution of galaxies within $100 \mathrm{Mpc}$}

Explosions of supernovae and mergings of binary massive compact objects are very rare in our Galaxy. Hence, only observations of many galaxies are expected to yield a reasonable detection rate.

From the Lyon-Meudon extragalactic database LEDA we extracted a sample of 33557 nearby galaxies within $100 \mathrm{Mpc}$. The 2D-distribution is shown on a Flamsteed equal area projection (Fig. 1). Some prominent structures appear. What are their distances? What is the actual space density of galaxies?

\subsection{The problem of the determination of distances}

The direct determination of distance requires difficult measurements and is thus available only for relatively small samples (5000 galaxies). The most efficient method consists of using the radial velocity together with a given Hubble constant. However, the determination of the Hubble constant is still controversial. Most of the difficulties come from the treatment of statistical biases. From the most recent absolute calibration and the best unbiased determination we adopt:

$H_{0}=60 \pm 5 \mathrm{~km} \mathrm{~s}^{-1} \mathrm{Mpc}^{-1}$.

Nevertheless, we must consider that some results are still suggestive of a larger value $\left(70 \pm 5 \mathrm{~km} \mathrm{~s}^{-1} \mathrm{Mpc}^{-1}\right)$, and even $H_{0}=50 \mathrm{~km} \mathrm{~s}^{-1} \mathrm{Mpc}^{-1}$ cannot be excluded. The smaller the value, the more difficult is GW detection due to larger distances to GW sources.

\subsection{The problem of spatial distribution}

Analysis of the 3D-galaxy distribution from the correlation function method (Davis \& Peebles 1983; Davis 1997) leads to the conclusion that the characteristic correlation length is $l_{0} \approx 5 \mathrm{Mpc}$ and the maximum inhomogeneity scale $l_{\max } \approx 20 \mathrm{Mpc}$. However, a more general statistical method to study large-scale galaxy distribution has been recently developed (see the review by Sylos Labini et al. 1998). It is applicable to any distribution of matter without the assumption of homogeneity (which is required in the correlation function analysis). The new analysis (the so-called conditional density function approach) is actually taken from modern statistical physics where it works as a standard tool.

Application of the conditional density function analysis to available redshift surveys of galaxies, such as CfA, SSRS, Perseus- Pisces, IRAS, LCRS etc., has revealed the fractal structure of the galaxy distribution up to the scales corresponding to the depth of these catalogs, i.e. about $100 \mathrm{Mpc}$ (see e.g. Pietronero et al. 1997; Sylos Labini et al. 1998). The fractal dimension of the spatial distribution is close to $\mathcal{D} \approx 2$. From the KLUN galaxy survey (Teerikorpi et al. 1998) where distances to galaxies are obtained by the Tully-Fisher method, it was shown that the observed number-distance relationship corresponds to a fractal dimension $\mathcal{D} \approx 2.2$ and continues up to the depth of the KLUN catalog, i.e. $200 \mathrm{Mpc}$.

The fractality implies that around any galaxy (including our own Galaxy) the density decreases as $r^{\mathcal{D}-3}$. This means that the number of galaxies does not increase as $r^{3}$ but rather as $r^{\mathcal{D}}$. The direct consequence for the present analysis is that the detection rate of GW events will be lower than previously thought.

From our sample we plotted the cumulative curves $\log N$ vs. $\log r$ ( $N$ is the total number of observed galaxies) within the radius $r(\mathrm{Mpc})$ (Fig. 2). This is done for different absolute magnitudes. Intrinsically faint galaxies $(M=-17)$ start to be missed beyond $\log r=1.3$ ( $\approx 20 \mathrm{Mpc}$ ), while galaxies brighter than $M=-22$ are observed up to the limit of our sample $(\approx 100 \mathrm{Mpc})$. The observed growth-curves correspond to $\mathcal{D} \approx 2.5$ (dashed curve in Fig. 2). They are used to calculate the correction allowing us to estimate the true number of galaxies in each direction, from the observed number. At a given distance, this correcting factor is simply deduced from the ratio of the observed and expected population (assuming $\mathcal{D} \approx 2.5)$. It is to be noted that up to $\log r=1.3$, even the faintest galaxies $(M=-17)$ follow the linear curve. This means that the sample is complete up to this distance (20 Mpc).

\section{Extragalactic sources of $\mathrm{GW}$ and intrinsic rates of events}

There are many sources of gravitational radiation in a galaxy. In fact, any accelerated motion generates GW. Among those usually discussed in the literature, galactic sources of GW are: supernova explosions, coalescing binary systems, binary stars, rotating asymmetric pulsars, active galactic nuclei. We consider only the GW sources which are expected to be sufficiently frequent and efficient to be detected in the near future.

The most powerful sources of gravitational radiation are the core-collapse supernovae (types Ib and II), merging neutron stars (ns), and black holes or other relativistic compact massive objects (cmo) (Thorne 1987, 1997) and also supernovae of type Ia, which are probably due to the explosion of a $\mathrm{CO}$ white dwarf and might be sufficiently strong candidates.

The relative supernova rates $R_{\mathrm{g}, \mathrm{s}}$ for galaxy type "g" and SN type "s" are free parameters in our code. For further calculations we adopt these from van den Bergh \& Tammann (1991), measured in SNU which equals one event per 100 yr per $10^{10} L_{0}^{B}$. In Table 1 we give the adopted rates of $\mathrm{SN}$ events for different morphological 


\section{Supergalactic coordinates}

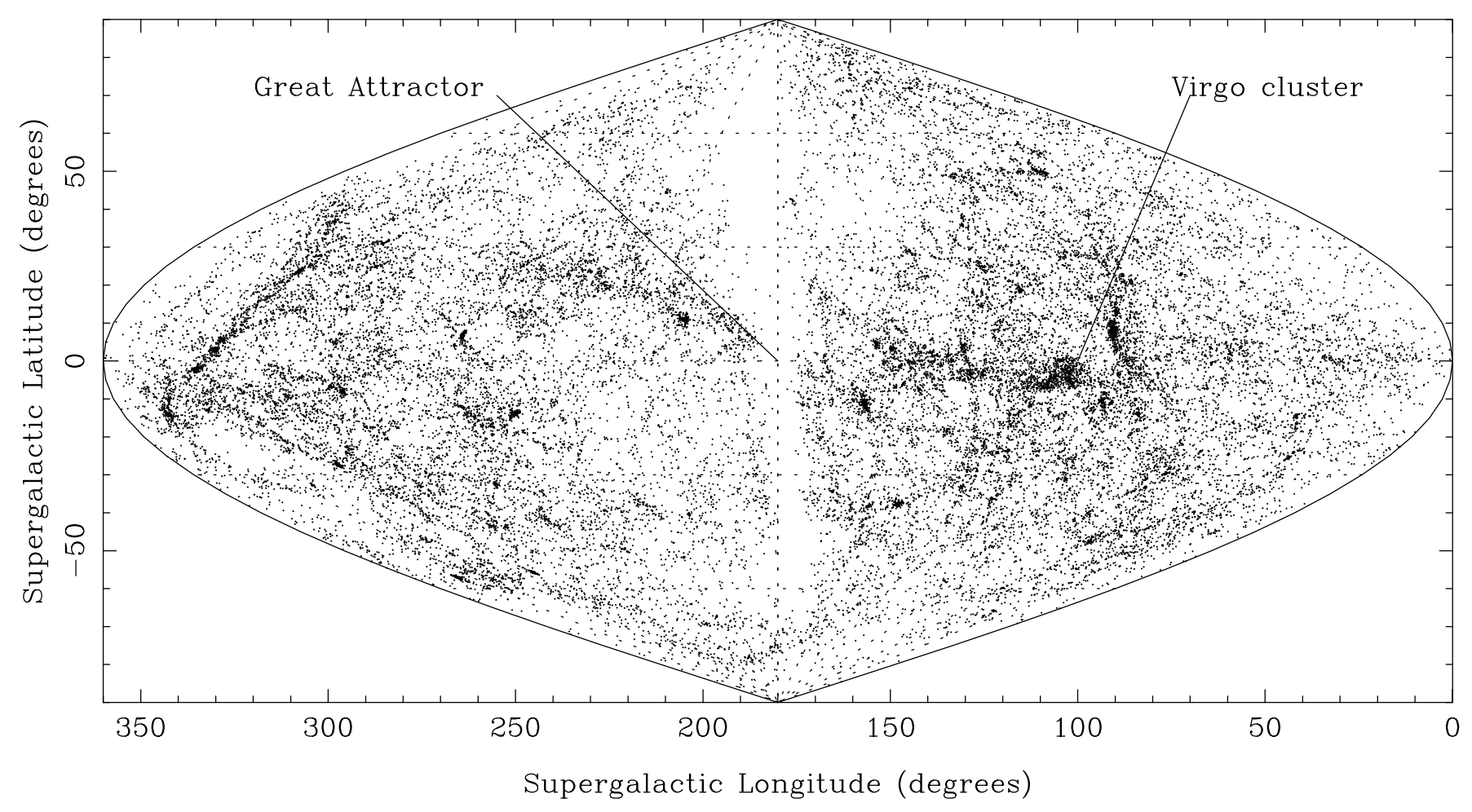

Fig. 1. Flamsteed equal area projection of our sample of 33557 galaxies located within $100 \mathrm{Mpc}$

Table 1. Adopted intrinsic rate of GW events. We consider Supernovae explosions (SNIa, SNIb, SNII) and coalescences of White Dwarfs (WD), Neutron Stars (NS), and Black Holes or Compact Massive Objects (CMO). The rate may depend on the morphological type of the parent galaxy (Elliptical(E), Spirals(S) or Irregulars(Irr))

\begin{tabular}{lc}
\hline source & $\begin{array}{c}\text { Rate } R_{\mathrm{g}, \mathrm{s}} \text { of GW events } \\
\text { per } 100 \text { years and } 10^{10} L_{\odot}\end{array}$ \\
\hline SNIa & $1.0(\mathrm{E}) 0.5(\mathrm{~S}$ and Irr) \\
SNIb & $0.0(\mathrm{E}) 0.3(\mathrm{~S}) 0.9$ (Irr) \\
SNII & $0.0(\mathrm{E}) 1.4(\mathrm{~S}) 4.2(\mathrm{Irr})$ \\
WD-WD & $310^{-2}$ \\
NS-NS & $310^{-3}$ \\
CMO-CMO & $10^{-3}$ \\
\hline
\end{tabular}

types of galaxies. Note that we use the Hubble constant $60 \mathrm{~km} \mathrm{~s}^{-1} \mathrm{Mpc}^{-1}$.

The event rate $R_{\text {merg }}$ for coalescing compact binaries composed of ns (or cmo) is still widely discussed and has a large uncertainty (e.g. Lipunov et al. 1997; PortegiesZwart \& McMillan 1999; Kalogera 1999). Here we adopt the values from Lipunov et al. (1995), however these events give a small contribution to the total statistics.

The first detailed study of the gravitational wave sky produced by galaxies within $50 \mathrm{Mpc}$ was done by Lipunov et al. (1995). They considered a wide class of GW sources in galaxies and used Tully's Nearby Galaxies Catalog comprising 2367 galaxies. In this paper we use 33557 galaxies from the LEDA catalogue and consider both tensor and scalar GW from supernova explosions.

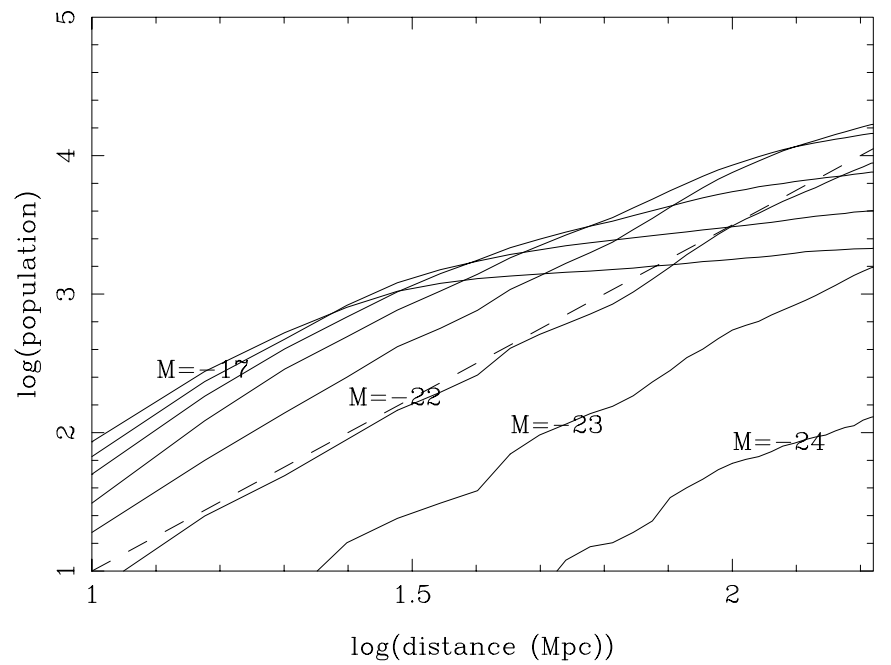

Fig. 2. Cumulative curves drawn for a wide range of absolute magnitudes (from $M=-24$ to $M=-17$ ). The completeness is severely affected for the less luminous galaxies $(M=-17)$ when the distance increases. The dashed curve shows the linear trend expected for a fractal dimension 2.5. These curves are used to derive the true space density of galaxies

\section{Theoretical amplitudes expected for GW events from SN}

\subsection{The problem of supernova explosion}

Expected amplitudes and forms of GW signals from supernova explosions detected on the Earth by gravitational detectors essentially depend on the adopted scenario of 
core-collapsed explosion of massive stars and relativistic gravity theory. This is why the forthcoming GW astronomy will give for the first time experimental limits on possible theoretical models of gravitational collapse including the strong field regime and even quantum nature of the gravity force.

For the estimates of the energy, frequency and duration of supernova GW emission one needs a realistic theory of SN explosion which can explain the observed ejection of the massive envelope. Unfortunately, for the most interesting case of SNII explosion such a theory does not exist now. As was recently noted by Paczynski (1999), if there were no observations of SNII it would be impossible to predict them from first principles.

Modern theories of the core collapse supernova are able to explain all stages of evolution of a massive star before and after the explosion. However, the theory of the explosion itself, which includes the relativistic stage of collapse where a relativistic gravity theory should be applied for the calculation of gravitational radiation, is still controversial and unable to explain the mechanism by which the accretion shock is revitalized into a supernova explosion (see the discussion by Paczynski 1999; Burrows 2000).

Moreover, recent observations of the polarization of core collapse supernovae (Wang et al. 1999) and the relativistic jet in SN1987A (Nisenson \& Papaliolios 1999) give strong evidence in favor of a jet-induced explosion mechanism for massive supernovae (Khokhlov et al. 1999; MacFadyen \& Woosley 1999; Wheeler et al. 2000). Further evidence for highly asymmetric SN explosions comes from recent observations of afterglows and host galaxies of gamma-ray bursts (Paczynsky 1999). This means that new "non-standard" scenarios of SN explosions (and hence GW radiation) may appear in the future and it may become important to study different possibilities for the expected GW signal (hence a wide range of GW parameters). Here we adopt scenarios existing in the literature to estimate the GW signal and we postpone the discussion of non-standard possibilities to Sect. 6 .

\subsection{Standard pulses of gravitational radiation}

The main aim of the present paper was not to provide realistic supernova explosion models, but to study the statistics of GW signals which may be expected in standard SN explosion scenarios. Hence we do not enter into the detailed calculations of the precise forms of GW signals within different gravity theories, but will simply use general energy arguments.

In our calculations we use the standard pulse of gravitational radiation introduced by Amaldi \& Pizzella (1979), which is a GW burst of sinusoidal wave with amplitude $h_{0}$, frequency $\nu_{0}$ and duration $\tau_{\mathrm{g}}$. For the case of tensor GW, the amplitude $h_{0}$ of the signal on the Earth due to the GW burst that occurs at a distance $r$ with total energy
$E_{\text {gw }}$ is (see e.g. Pizzella 1989):

$$
\begin{aligned}
& h_{0}=1.4 10^{-20}\left(\frac{1 \mathrm{Mpc}}{r}\right)\left(\frac{1 \mathrm{kHz}}{\nu_{0}}\right) \\
& \times\left(\frac{E_{\mathrm{gw}}}{1 M_{\odot} c^{2}}\right)^{1 / 2}\left(\frac{1 \mathrm{~s}}{\tau_{\mathrm{g}}}\right)^{1 / 2} .
\end{aligned}
$$

For the case of scalar GW the amplitude $h_{0}$ is given by the same relation with a pre-factor depending on the considered theory, e.g. for tensor field gravity theory it is $\approx 210^{-20}$ (Baryshev 1995).

Hence, each type of GW source at a fixed distance $r$ is characterized by three main observable parameters $E_{\mathrm{gw}}$, $\nu_{0}$ and $\tau_{\mathrm{g}}$. In the next subsection we choose acceptable values for these parameters.

\subsection{Adopted values for energy, frequency and duration of tensor and scalar GW pulses}

Let us first consider the parameters for standard tensor GW pulses in General Relativity. There is no unique widely accepted model for tensor GW radiation produced by SN core collapse and in the literature two main scenarios are usually discussed: axisymmetric and nonaxisymmetric ones, reviewed by Thorne (1997).

Within the theory of axisymmetric rotational core collapse, Zwerger \& Muller (1997) found that the energy spectrum covers a frequency $50 \mathrm{~Hz}<\nu_{0}<3 \mathrm{kHz}$ but most of the power is emitted between $500 \mathrm{~Hz}$ and $1 \mathrm{kH}$. Duration of the pulses lies between $0.5-5 \mathrm{~ms}$. According to numerical calculations by Stark \& Piran (1985), considered in Ferrari et al. (1999) as a basis for prediction of GW background from SN, the GW energy spectrum has two maxima around $5 \mathrm{kHz}$ and $9 \mathrm{kHz}$. The duration of $\mathrm{GW}$ pulses also is of the order a few ms. In accordance with these calculations, for our statistical approach, we adopt the characteristic frequency $\nu_{0}=1 \mathrm{kHz}$ and the duration of the pulse $\tau_{\mathrm{g}}=1 \mathrm{~ms}$.

For the total GW energy radiated by SN core collapse there is a very large range of predicted values in the literature. According to Zwerger \& Muller (1997) the energy radiated in the form of GWs lies in the range $610^{-11} M_{\odot} c^{2}<E_{\mathrm{gw}}<810^{-8} M_{\odot} c^{2}$, which is consistent with Bonazzola \& Marck's (1993) results for the deformation parameter $s<0.1$. However fully relativistic numerical simulations by Stark \& Piran, also adopted by Ferrari et al. (1999), give $E_{\mathrm{gw}} \leq 10^{-3} M_{\odot} c^{2}$. Moreover, if the collapsing core rotates so rapidly that it becomes nonaxisymmetric and may be transformed into a bar-like configuration, which also might break up into several fragments, then the GW radiation could be almost as strong as that from a coalescing neutron star binary. Several specific scenarios for such nonaxisymmetric SN core collapses have been proposed (see review by Thorne 1997). According to Lai \& Shapiro (1995) the energy radiated in GW during the nonaxisymmetric stage of the gravitational collapse can be as large as $410^{-3} M_{\odot} c^{2}$. Bonnell \& Pringle (1995) considered gravitational radiation from SN core collapse 
and fragmentation, which could produce the GW energy $10^{-2} M_{\odot} c^{2}$. For our statistical study of GW events we choose, as a basis, the value $E_{\mathrm{gw}}=10^{-6} M_{\odot} c^{2}$.

Within classical general relativity there is no scalar GW and for instance spherically symmetric collapse does not generate gravitational radiation. However other relativistic and quantum gravity theories predict both tensor and scalar GW. Calculations of amplitudes, frequencies and forms of the scalar gravitational radiation in the case of spherically symmetric SN core collapse has been made by Shibata et al. (1994), Harada et al. (1997), Novak \& Ibanez (1999). The released scalar GW energy is of order $\omega^{-1} M_{\odot} c^{2}$, where $\omega \geq 10^{3}$ is the parameter of the BransDicke theory. The characteristic frequency and duration are similar to the tensor GW in general relativity. For the comparison of the statistics of tensor and scalar events we adopt the same energy, frequency and duration for both scalar and tensor GW's.

In Fig. 3 we plot the expected amplitudes of GWs calculated according to Eq. (2) for a wide range of the parameter $E_{\mathrm{gw}}$, which covered the expected values of GW energy for tensor and scalar GW. Three pairs of lines $(\mathrm{a}, \mathrm{b}, \mathrm{c})$ correspond to the following combinations of the main GW pulse parameters:

a) $E_{\mathrm{gw}}=10^{-3} M_{\odot} c^{2} ; \nu_{0}=10^{3} \mathrm{~Hz} ; \tau_{\mathrm{g}}=1 \mathrm{~ms}$;

b) $E_{\mathrm{gw}}=10^{-6} M_{\odot} c^{2} ; \nu_{0}=10^{3} \mathrm{~Hz} ; \tau_{\mathrm{g}}=1 \mathrm{~ms}$;

c) $E_{\mathrm{gw}}=10^{-9} M_{\odot} c^{2} ; \nu_{0}=10^{3} \mathrm{~Hz} ; \tau_{\mathrm{g}}=1 \mathrm{~ms}$.

For further calculations we adopt the values of GW amplitudes which correspond to the case $b$. Obviously, our calculations may be rescaled by using any other combinations of main GW parameters according to Eq. (2).

In Fig. 3 we draw two levels of sensitivity (horizontal dotted lines) which can be expected today $\left(h_{0}=10^{-21}\right.$ and $\left.10^{-22}\right)$. One sees that it would be possible to detect SN explosions at the distance of the Virgo cluster and of the Great Attractor only with an optimistic energy release (case $a)$.

\subsection{Longitudinal and transversal scalar GW}

An important difference between tensor and scalar GWs is that tensor waves (spin 2) are transversal while scalar waves (spin 0) can be transversal and/or longitudinal. There is no scalar GW in general relativity.

In the frame of the Jordan-Fierz-Brans-Dicke theory, as for any metric tensor-scalar theories, the scalar wave is transversal but isotropic in the plane transversal to the propagation direction (Damour \& Esposito-Farese 1992). The transversal spin 0 wave may be presented as the metric perturbation in the following form (Bianchi et al. 1998; Brunetti et al. 1999; Maggiore \& Nicolis 2000):

$g_{i k}=h_{0} \cos (\omega t-k z) \operatorname{diag}(0,1,1,0)$.

In the frame of the field approach to gravity formulated in 60's by Feynman (see e.g. Feynman et al. 1995;

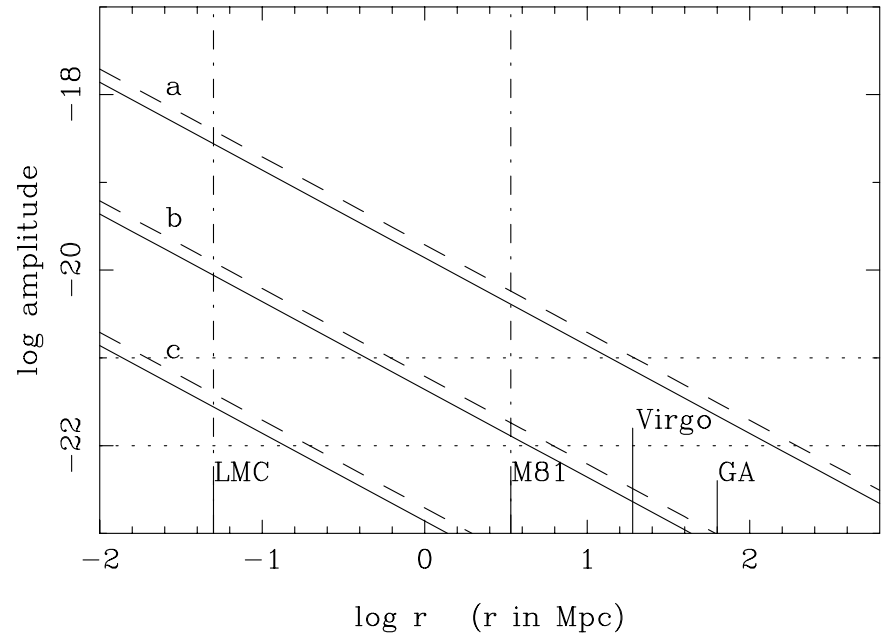

Fig. 3. Theoretical GW amplitude versus distance. The predicted amplitude at a distance $r$ is given for tensor (solid lines) and scalar (dashed curves) waves according to Eq. (2) for cases $\mathrm{a}, \mathrm{b}, \mathrm{c}$ (see text). For instance, case $b$ corresponds to the GW energy of $10^{-6} M_{\odot} c^{2}$, a pulse duration $\tau_{\mathrm{g}}=10^{-3} \mathrm{~s}$ and a frequency $\nu_{0}=1 \mathrm{kHz}$. Two sensitivity levels are represented (dotted lines) corresponding to $h_{0}=10^{-21}$ and $10^{-22}$. The distances of LMC, M 81, Virgo and Great Attractor are labelled on the $x$-axis

Thirring 1961; Straumann 2000), there are also scalar gravitational waves which are longitudinal and generated by the trace of the energy-momentum tensor (Baryshev 1982, 1995; Sokolov 1992). In such a scalar wave, a test particle moves along the direction of the wave propagation. The most straightforward way to demonstrate this is to use exact relativistic equations of motion of test particles in tensor and scalar potentials derived by Kalman (1961). It is an important fact that the physical interaction of a GW with a detector may be completely analyzed in terms of the weak field approximation, i.e. with the usual Lagrangian formalism of relativistic field theory in Minkowski space (see Appendix).

The scalar plane monochromatic GW in the system of coordinates with the $z$-axis directed along the wave propagation may be presented in the form:

$h_{i k}=h(t, z) \eta_{i k}$

where $h(t, z)$ is a 4 -scalar field of Minkowski space, or

$h_{i k}=h_{0} \cos (\omega t-k z) \operatorname{diag}(1,-1,-1,-1)$

where $h_{0} \ll c^{2}$ is the amplitude of the wave, $\omega=2 \pi \nu_{0}, k=$ $\omega / c=2 \pi / \lambda, \eta_{i k}=\operatorname{diag}(1,-1,-1,-1)$ is the Minkowski metric tensor. As shown in the Appendix the test particles in such a gravitational potential are moving in the direction of the wave propagation ( $z$-axis). The analysis of the motion of test particles is sufficient for the description of an interaction of the scalar GW with interferometric and bar detectors because the scalar GW does not interact with electromagnetic field. Indeed, the interaction 
Lagrangian is $\Lambda_{\mathrm{int}}=h_{i k} T_{\mathrm{em}}^{i k}=0$ due to the tracelessness of the energy-momentum tensor of the electromagnetic field. Hence without any ambiguity, the scalar GW is longitudinal in the field gravity theory.

This means that both types of scalar GWs, longitudinal and transversal, are theoretically possible and physically different. The physical difference between longitudinal and transversal scalar GW may be experimentally established by comparing the direction of the maximum sensitivity of a bar detector with the direction of the axis of the bar. Indeed, for a bar detector the maximum sensitivity to the transversal GW is in the direction orthogonal to the bar axis, while for the longitudinal scalar GW the maximum sensitivity direction is along the axis of the bar.

For an interferometric detector (which has two arms) the maximum sensitivity to the tensor GW will be in the direction orthogonal to the plane containing both arms. For the longitudinal scalar waves there are two directions of maximum sensitivity which coincide with the directions of each arm. It is interesting that for the transversal scalar GW the directions of maximum sensitivity also coincide with the two arms of the interferometer (Maggiore \& Nicolis 2000; Nakao et al. 2000). This is a special case of a two arm interferometer, while in the case of a bar detector the detector patterns are different for longitudinal and transversal scalar GWs.

The difference in the response of GW detectors to arriving tensor and scalar GW pulses allows one to test the nature of the detected waves. Below we compare the statistics of expected GW events for transversal and longitudinal GWs.

\section{Probability of GW events as a function of sidereal time}

\subsection{The expression for the number of GW events}

Let us consider a sample of galaxies. Each galaxy may produce GW events with a certain rate. These events will be either detected or not, depending on the amplitude of the signal and on the sensitivity of the detector. The number of detected events produced at a time $t$ for a given type of wave (tensor or scalar noted by index $w$ ) and a given detector (interferometer or bar detector noted by index $d$ ) will be the sum of the contribution of each galaxy to the considered type of source (SNIa, SNIb, SNII etc. noted by index $s$ ).

$\frac{\mathrm{d} P}{\mathrm{~d} t}(t, s, w, d)=\sum_{g} L_{g} \times R_{g, s} \times \Delta$

where $L_{g}$ is the luminosity (in $L_{\odot}$ ) of the $g$ th galaxy; $R$ is the rate of GW events per year and per $L_{\odot}$ for the considered type of source and the considered type of wave; $\Delta$ is the detectability calculated from the observed amplitude $h_{\text {obs }}$ and the limiting amplitude $h_{\lim }$ of the detector.

Let us detail the three terms used in Eq. (6).
- The luminosity $L_{g}$ is derived from the absolute blue magnitude $M_{g}$ of the $g$ th galaxy. A correction for incompleteness is done using Fig. 2 and assuming that missed galaxies are distributed like the observed ones. This does not account for structures completely hidden by the disk of our Milky Way ${ }^{1}$. The luminosity is:

$L_{g}=C\left(M_{g}, r_{g}\right) 10^{-0.4\left(M_{g}-M_{\odot}\right)}$

$\left(r_{g}\right.$ is the distance of the $g$ th galaxy and $M_{\odot}=5.2$ is the absolute magnitude of the Sun in the photometric $B$-band) From Fig. 2 it is visible that the correcting term $C$ is almost equal to one below $20 \mathrm{Mpc}$ $(\log r=1.3)$, because all curves are linear even for the faintest galaxies. This means that up to the Virgo cluster $(\approx 18 \mathrm{Mpc})$ all galaxies are included;

- The rate $R_{g, s}$ depends on the morphological type of the considered galaxy (index $g$ ) and on the type of the GW source (index $s$ ) according to Table 1 . As an example, we made calculations for SNII + SNIb. Hence, the expected counts will be underestimated, if all other conditions are satisfied;

- The detectability $\Delta$ is defined as:

$\Delta= \begin{cases}1 & \text { if } h_{\mathrm{obs}}>h_{\mathrm{lim}}, \\ 0 & \text { if } h_{\mathrm{obs}}<h_{\mathrm{lim}} .\end{cases}$

We will consider a sensitivity of $h_{\lim }=10^{-23}$. It is not yet accessible by present and scheduled detectors, but one can hope an improvement will be possible from space missions. The observed amplitude is defined as:

$h_{\mathrm{obs}}=h_{\mathrm{o}} \cdot|G(\alpha, \delta, w, d)|$

$h_{\mathrm{o}}$ is given by Eq. (2). It depends on the considered type of source (via the energy, frequency and duration of the pulse) and on the considered type of gravitational wave. $G(\alpha, \delta, w, d)$ is the geometrical factor. It characterizes the relative orientation of the GW and of the detector at the time of the observation. It depends on the type and position of the detector (characterized by its latitude, sidereal time and azimuth of its reference axis), on the direction of the GW (characterized by the equatorial coordinates $\alpha, \delta$ of the $g$ th galaxy), and on the type of wave (including polarization for tensor waves). Note that the longitude of the site is not needed explicitly because it is included in the definition of the sidereal time. The sidereal time will be given in hours (from 0 to 24 ).

The calculation of the geometrical factor $G$ is explained in the next subsection for interferometric and bar detectors.

\subsection{Geometry of the system}

The geometrical configuration is defined by the wave direction $(O S)$, the local horizon (perpendicular to $O Z$, where $Z$ is the zenith) and the equatorial plane (perpendicular to $O P$, where $P$ is the equatorial northern pole). The main features of the system are shown in Fig. 4.

\footnotetext{
${ }^{1}$ For example, the Great Attractor which is presented in Sect. 5.2
} 


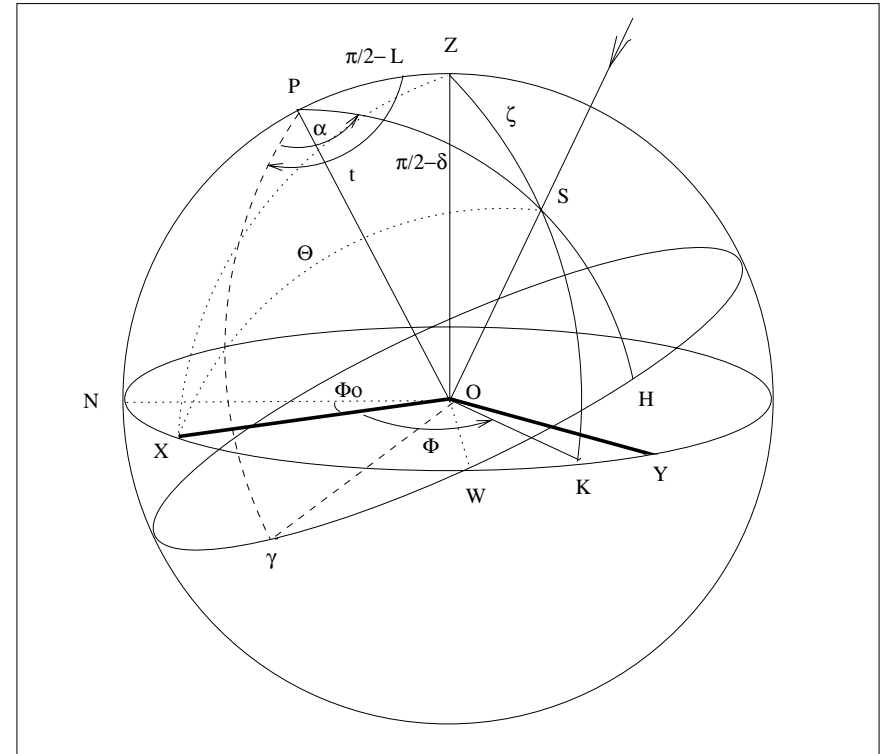

Fig. 4. The main geometrical definitions. $Z$ is the zenith of the site. $P$ is the northern pole of the equatorial coordinate system. $\gamma$ defines the sidereal time with respect to the southern meridian. The source $S$ is located by its equatorial coordinates $\alpha=\widehat{\gamma O H}$ for the right ascension and $\delta=\widehat{H O S}$ for the declination. The distance to the zenith is $\zeta=\widehat{Z O S}$. The reference direction for the detector is the direction $O X$. It is either the $x$-arm for an interferometric detector or the direction of the bar for a bar detector. The azimuth of this direction is $\Phi_{\mathrm{o}}$. It is defined in the direct sense from the north to the west

The expression of the geometric factor for tensor waves has been calculated by Schutz \& Tinto (1987) and Thorn (1987). For scalar waves it has been calculated by Baryshev (1997).

The relevant angles are $\zeta, \Phi, \Theta$ and $\Psi$. They depend on the different cases. Let us detail:

- For interferometric detectors: the zenith distance $\zeta=$ $\widehat{Z O S}$ (angle between the direction of the zenith and the arrival-direction of the wave), the azimuth $\Phi=\widehat{X O K}$ of the direction of the wave (direction OK) calculated with respect to the reference axis of the detector;

- For bar detectors: only the angle $\Theta=\widehat{X O S}$ is relevant;

- For tensor GW the polarization angle $\Psi$. As in Schutz \& Tinto (1987) it may be measured in the GW plane from the line of nodes (intersection of the GW plane and of the horizontal plane).

Note that the direction of the polarization $\Psi$ depends on the geometry of the emitting source. For the calculation with the actual galaxy distribution $\Psi$ will be simply chosen at random. With these notations we obtain the following results: $\zeta$ and $\Phi$ are calculated by solving the spherical triangle $Z P S$. One obtains:

$\cos \zeta=\sin L \sin \delta+\cos L \cos \delta \cos (t-\alpha)$

$L$ is the latitude of the site where the detector operates. $\alpha$ and $\delta$ are the equatorial coordinates of the source, and $t$ is the sidereal time at the site. $\zeta$ is defined in the range $(0, \pi)$.

$$
\begin{aligned}
& \cos \left(\Phi_{\mathrm{o}}+\Phi\right)=\frac{\cos L \sin \delta-\sin L \cos \delta \cos (t-\alpha)}{\sqrt{1-\cos ^{2} \zeta}} \\
& \sin \left(\Phi_{\mathrm{o}}+\Phi\right)=\frac{\cos \delta \sin (t-\alpha)}{\sqrt{1-\cos ^{2} \zeta}}
\end{aligned}
$$

$\Phi_{\mathrm{O}}$ is the azimuth of the reference axis of the system counted in the direct sense from the direction of the north. In this paper the reference axis is $O X$, as in Thorn (1987). It is either the direction of the $\mathrm{X}$-arm for an interferometric detector or the direction of the bar for a bar detector. $\Phi$ is defined over the range $(0,2 \pi)$.

The angle $\Theta$ is calculated by solving the triangle $X Z S$ :

$\cos \Theta=\sin \zeta \cos \Phi$

$\Theta$ is defined over the range $(0, \pi)$.

The relevant angles being calculated according to the previous relations, the geometrical factor in the four considered cases has the following form:

1. For tensor waves and interferometric detector the geometrical factor is:

$G=0.5\left(1+\cos ^{2} \zeta\right) \cos 2 \Phi \sin 2 \Psi-\cos \zeta \sin 2 \Phi \cos 2 \Psi$.

2. For longitudinal scalar waves and interferometric detector the geometrical factor is:

$G=\sin \zeta(|\cos \Phi|-|\sin \Phi|)$.

3. For tensor waves and bar detector the geometrical factor is:

$G=\sin ^{2} \Theta \cos 2 \Psi$

4. For longitudinal scalar waves and bar detector the geometrical factor is (Appendix, Eq. (A.22)):

$G=\cos \Theta$.

The interferometric GW detectors (such as TAMA, GEO600, VIRGO, LIGO) have the frequency range of about $10-1000 \mathrm{~Hz}$ and the sensitivity $h \approx 10^{-20}-10^{-22}$. Sensitivity is a measure of the detectable amplitude and it is proportional to $\Delta l / l$, the relative length variation of the arms of the interferometer.

Presently working bar detectors, such as IGEC RBO (International Gravitational Event Collaboration of Resonant Bar Observatory) which includes five cryogenic resonant bar detectors (ALLEGRO, AURIGA, EXPLORER, NAUTILUS, NIOBE), have a typical bandwidth of the order of $1 \mathrm{~Hz}$ around each one of the two resonances (close to $1000 \mathrm{~Hz}$ ). The achieved sensitivity is now $F_{0} \approx 2-610^{-21} \mathrm{~Hz}^{-1}$ (Prodi et al. 2000). In future one expects the sensitivity to be at the level of $10^{-22} \mathrm{~Hz}^{-1}$.

We have calculated the predicted amplitude for different existing detectors and specific galaxy clusters. The closest concentration of galaxies is the Virgo cluster. 
The name of the VIRGO detector comes from the name of the cluster itself because it may be the main source of first detectable GW events. The Virgo cluster is not very far from our Local Group (20 Mpc). It induces a velocity of about $170 \mathrm{~km} \mathrm{~s}^{-1}$ on the Local Group. On the other hand, it has been claimed (Dressler et al. 1987) that a hidden large concentration of galaxies (hereafter, the Great Attractor) induces a velocity of about $500 \mathrm{~km} \mathrm{~s}^{-1}$ on our Local Group (i.e. about three times more). The distance of such a concentration has been estimated to be three times the distance to Virgo. This means that the number of galaxies could be about 27 times larger than the number of Virgo galaxies ${ }^{2}$. If the sensitivity of GW detectors is improved, the Great Attractor may become the major source of GW events. This justifies the interest we place in this region. The position of this putative Great Attractor would be roughly at galactic coordinates $l=310 \mathrm{deg}, b=0 \mathrm{deg}$. This is well supported by the apparent 2D-distribution of galaxies which shows that this region may constitute a link between two visible structures on both sides of the Milky way (Paturel et al. 1987 and Fig. 1) and by the discovery of a large number of galaxies around this region (Kraan-Korteweg 2000).

Then, we considered two clusters as dominant sources. The adopted equatorial coordinates and distances are the following:

\begin{tabular}{llll}
\hline Cluster & $\alpha(1950)$ & $\operatorname{delta}(1950)$ & $r$ \\
\hline Virgo & $12 \mathrm{~h} 28 \mathrm{~m}$ & $+12 \operatorname{deg} 40^{\prime}$ & $20 \mathrm{Mpc}$ \\
Great-Attractor & $15 \mathrm{~h} 00 \mathrm{~m}$ & $-60 \operatorname{deg} 00^{\prime}$ & $60 \mathrm{Mpc}$ \\
\hline
\end{tabular}

We considered the following detectors:

\begin{tabular}{llrl}
\hline Detector & Latitude $L$ & Azimuth $\Phi_{\mathrm{o}}$ & Type \\
\hline VIRGO & $44.7 \mathrm{deg}$ & $-15 \mathrm{deg}$ & interf. \\
AURIGA & $45.4 \mathrm{deg}$ & $+45 \mathrm{deg}$ & bar \\
NAUTILUS & $41.8 \mathrm{deg}$ & $0 \mathrm{deg}$ & $\mathrm{bar}$ \\
NAUTILUS & $41.8 \mathrm{deg}$ & $90 \mathrm{deg}$ & bar \\
\hline
\end{tabular}

In the following subsections we calculate the amplitudes in different conditions, for different detectors and sources. For the study of the polarization effect on tensor waves (Figs. 5 to 6 ) we used the cluster Virgo (as a point source) and detectors VIRGO and AURIGA. For the comparison of the distribution of the amplitudes of Virgo and of the Great-Attractor (Figs. 7 to 10) we used Virgo and the Great attractor as point sources and the detectors VIRGO, AURIGA and NAUTILUS. For the calculation of the density of probability of GW events along the sideral time (Figs. 11 and 12) we used the sample of individual galaxies and the detectors VIRGO, AURIGA and NAUTILUS.

${ }^{2}$ The velocity, and thus the acceleration during the same time, is three times larger. The distance is also three times larger, resulting in a mass nearly $3 / 3^{-2}=27$ times larger.
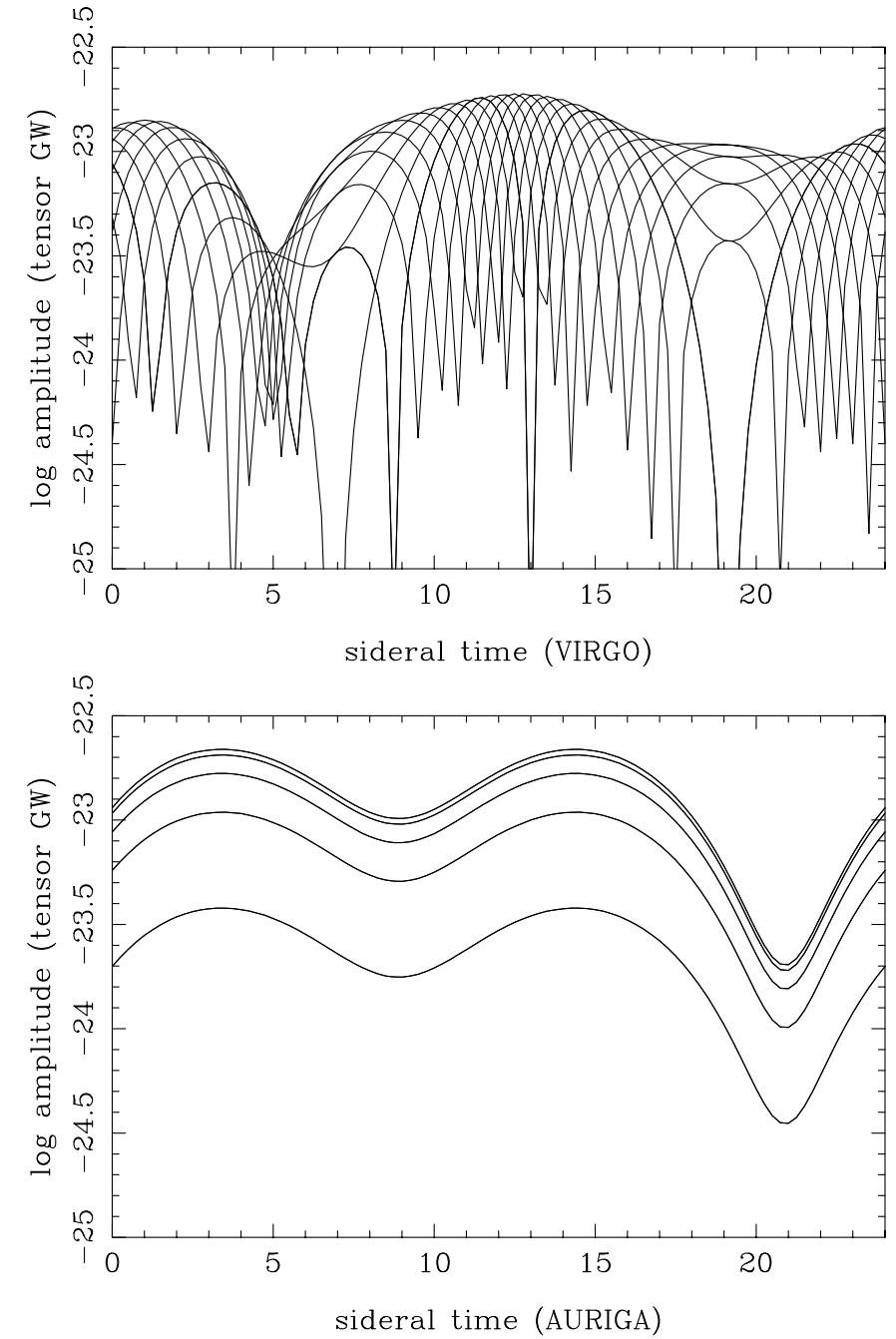

Fig. 5. a) Amplitude as a function of sidereal time for tensor GW emitted by Virgo and seen with the VIRGO interferometric detector for different polarizations of the GW. Each curve represents 36 fixed polarization directions. b) Amplitude as a function of sidereal time for tensor GW emitted by Virgo and seen with the AURIGA bar detector for different polarizations

\subsection{Effect of the polarization}

Because the polarization angle cannot be predicted, it is important to show the effect of the polarization for tensor waves. Considering only the Virgo cluster and interferometric (VIRGO) and bar detector (AURIGA), we calculated the amplitude according to Eq. (9) using $h_{o}$ for case b (Fig. 3). The geometrical factor is given by Eqs. (14) and (16), for an interferometric and bar detector respectively. We used 36 polarization angles over the range $(0,2 \pi)^{3}$. The results are shown in Figs. 5a,b, respectively.

For interferometric detectors, the different curves are shifted along the $x$-axis (sidereal time) depending on the polarization. On the contrary, for bar detectors, the curves are shifted along the $y$-axis (amplitude); hence, the

\footnotetext{
3 In fact, the period is $\pi / 2$ for interferometric detectors and $\pi / 4$ for bar detectors.
} 
minima and maxima always appear at the same $x$ values (same sidereal times). Let us explain why it is not correct to calculate the mean over the different polarizations. If an event is produced with the favorable polarization, it will be detected if the amplitude is larger than the limiting amplitude. On the other hand, if the polarization is unfavorable, the observed amplitude will be reduced and the considered event may fall below the limiting amplitude. The global effect of the uncertainty on the polarization is simply a reduction of the number of counted events, but not a reduction of the amplitude. Finally, the distribution of the amplitudes along the sidereal time will be simply given by the envelope of the curves obtained for the different polarizations. It must be noted that it is a handicap for interferometric detectors because the contrast is smoothed and the total number of expected events is reduced. We repeated the same calculations with polarizations taken at random (Figs. 6a,b). The same effect is clearly visible. The curves are the envelopes of the previous ones. Some events are seen with smaller amplitudes due to unfavorable polarization.

\subsection{Comparison of the amplitudes of GW from Virgo and the Great Attractor}

We have calculated the GW amplitudes as a function of sidereal time for the four detectors listed in the previous table. For each of them we give two figures, for tensor and scalar GW, respectively. In each figure we present the amplitudes expected for sources in the Virgo cluster (solid curve) and in the Great Attractor region (dashed curve).

The results are given in four figures (Figs. 7 to 10): The caption of each figure contains detailed comments. Here, we will simply highlight prominent features.

With a sensitivity $h_{\text {lim }}=10^{-23}$ only GW events from the Virgo cluster (solid lines) will be detectable. The Great Attractor (dashed curves) will be detectable only with a sensitivity of $h_{\lim }=10^{-23.5}$. Nevertheless, there is one case (VIRGO detector - Fig. 7b) where scalar waves could be seen from the Great Attractor with a sensitivity of $h_{\text {lim }}=10^{-23}$. Because we expect about 25 times more events from the Great Attractor, this may result in a peak in the rate of events around sidereal time $t=17 \mathrm{~h}$.

Another important features in these diagrams of amplitudes is that tensor and scalar waves give peaks which generally have opposite phases. In other words, if we expect a maximum of events for scalar waves, there should be a minimum for tensor waves. This is clearly visible by comparing Figs. 8a and b. This is also important because it may be used to disentangle the contributions of these two kinds of waves. The same characteristic is present when we compare the expected counts for the NAUTILUS bar detector with two perpendicular orientations $\left(\Phi_{\mathrm{O}}=0 \mathrm{deg}\right.$ and $\left.\Phi_{\mathrm{o}}=90 \mathrm{deg}\right)$. The figure suggests that NAUTILUS could benefit from an orientation complementary to the one used, e.g., with the AURIGA orientation.
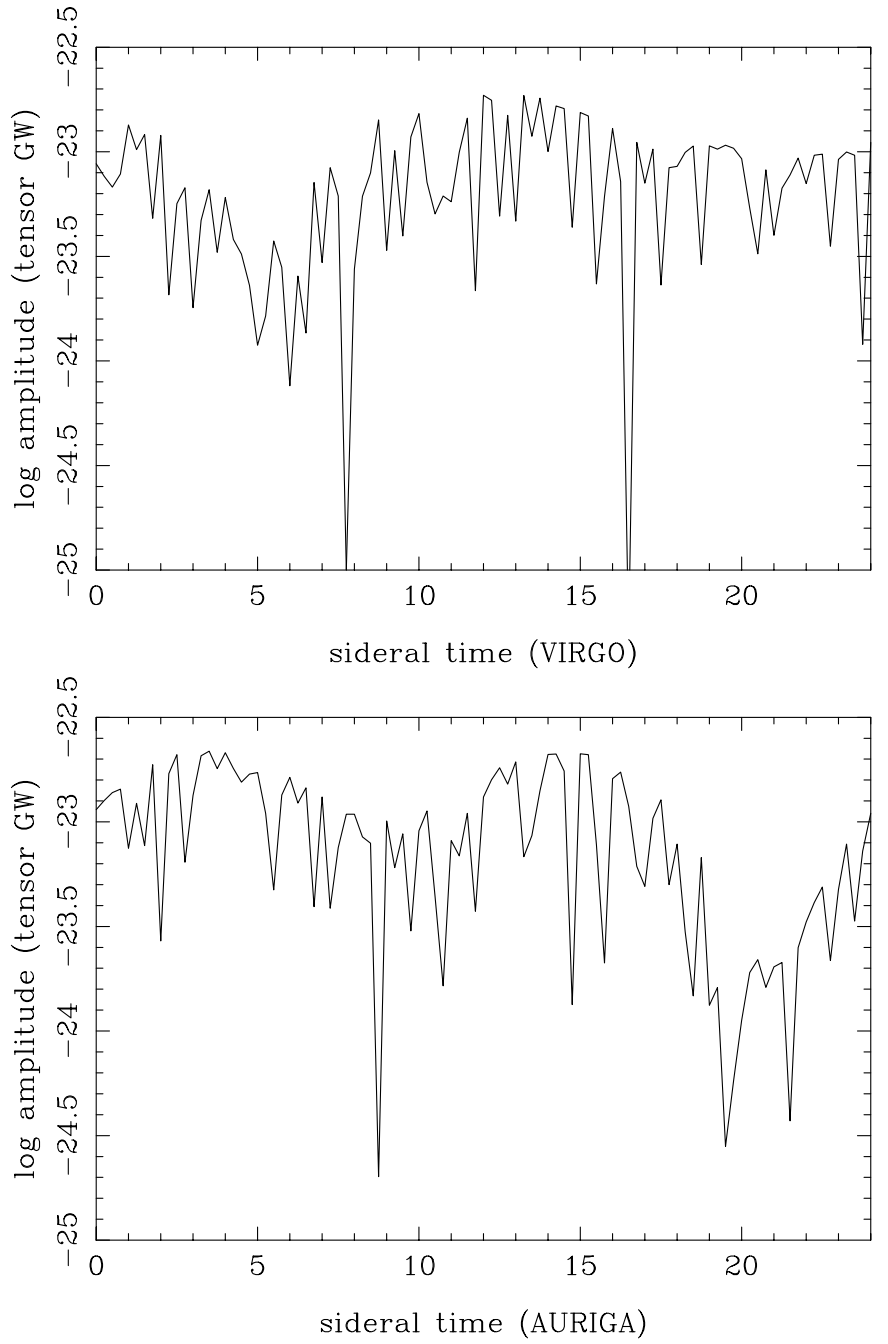

Fig. 6. a) Amplitude for the VIRGO detector as in Fig. 5a but with random directions of polarization. The shape is unchanged but some events have a reduced amplitude. This will lead to a reduction of the GW events detected at a given sensitivity level. b) Amplitude for the AURIGA detector as in Fig. $5 \mathrm{~b}$ but with random directions of polarization. The shape is unchanged but, on average, the amplitude is reduced

\subsection{Number of GW events expected for the actual galaxy distribution within $100 \mathrm{Mpc}$}

Using the catalog of galaxies described in Sect. 2, we simulated the count of GW events using Eq. (6). The calculation is made with an energy of $E_{\mathrm{gw}}=10^{-6} M_{\odot} c^{2}$, a frequency of $1 \mathrm{kHz}$ and a duration of $1 \mathrm{~ms}$. This corresponds to the case b of Fig. 3. The adopted limiting amplitude $h_{\lim }$ is $10^{-23}$. It should allow us to reach the Virgo cluster. The calculation is done for VIRGO, AURIGA, NAUTILUS (azimuth $=0 \mathrm{deg}$ ) and NAUTILUS (azimuth $=90 \mathrm{deg})$. We plotted simultaneously the counts for tensor (solid curves) and scalar waves (dashed curves). The results are shown in Figs. 11 and 12 for the four considered detectors. When comparing these figures with the expected amplitudes calculated for the detectors VIRGO and AURIGA, it is seen that all predicted maxima are 

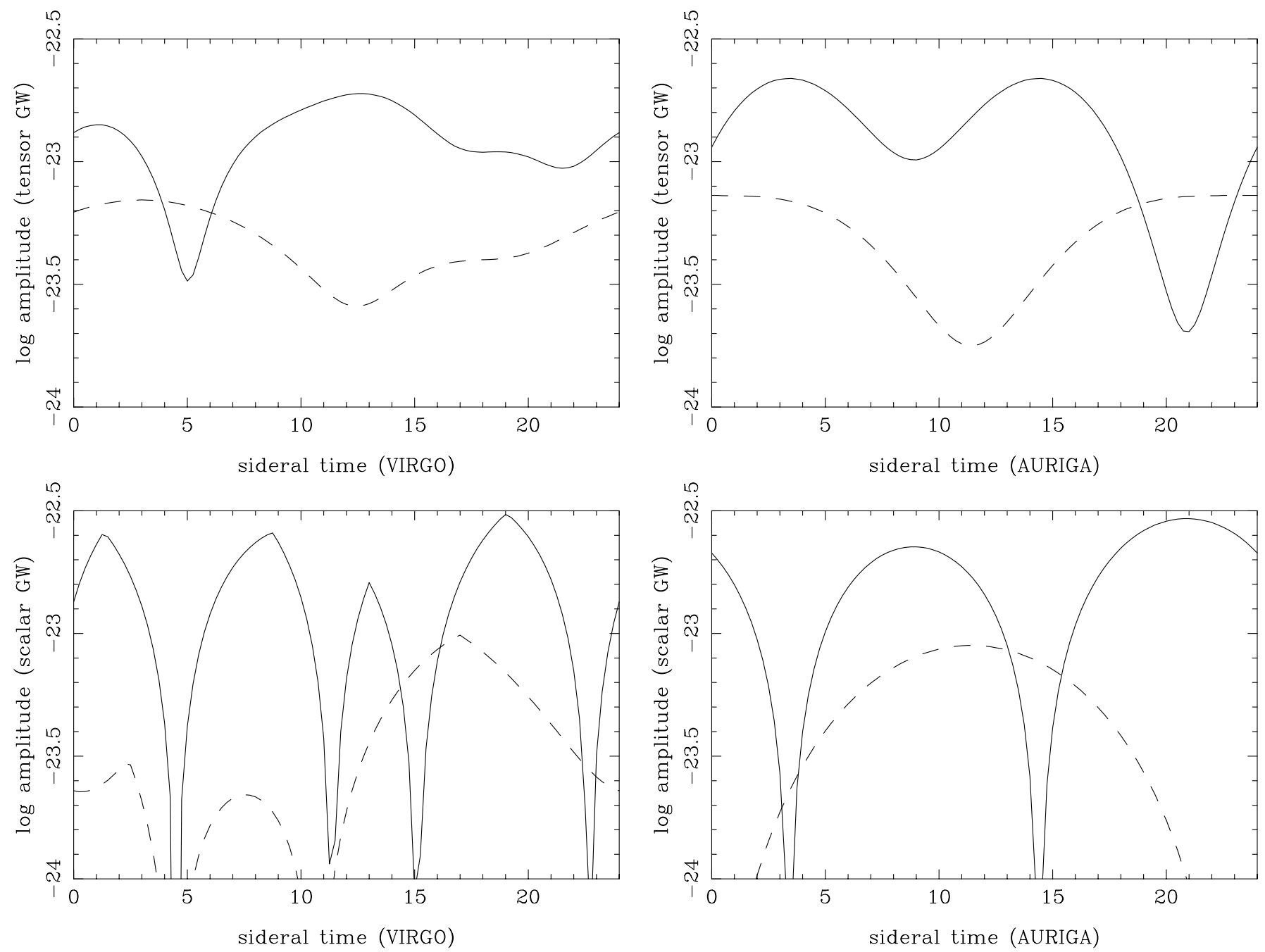

Fig. 7. a) Amplitude as a function of sidereal time for tensor GW emitted by Virgo (solid curve) and the Great Attractor (dashed curve) as seen with the interferometric VIRGO detector. With a sensitivity of $10^{-23}$ only Virgo will give large enough amplitude to make events detectable between $t=9 \mathrm{~h}$ and $t=15 \mathrm{~h}$ and between $23 \mathrm{~h}$ and $4 \mathrm{~h}$. The Great Attractor could be detected with a sensitivity of $10^{-23.5}$ (except between $t=11 \mathrm{~h}$ and $t=15 \mathrm{~h}$ ). b) Ibidem but for scalar waves. Virgo (solid curve) could be detectable at different peaks along the sidereal time. The Great Attractor could be barely detected with a sensitivity of $10^{-23}$ at $t=17 \mathrm{~h}$

at the expected positions according to the distribution of amplitudes given by the Virgo cluster alone (solid curves in Figs. $7 \mathrm{a}$ and $\mathrm{b}$ and $8 \mathrm{a}$ and b). This confirms that, within a distance of $20 \mathrm{Mpc}$, the Virgo cluster dominates. This was not obvious because the influence of other galaxies was not easy to predict. The expected number of GW events reaches a few tens per year at the most favorable sidereal time but with a yet unreachable sensitivity for the considered GW energy. For instance, from Fig. 11a we see that several maxima are expected for scalar waves (dotted curve) in agreement with their positions predicted from Fig. 7b for the Virgo cluster alone. Similarly, the maximum at $t \approx 13 \mathrm{~h}$ for tensor waves (solid curve) is the one

Fig. 8. a) Amplitude as a function of sidereal time for tensor GW emitted by Virgo (solid curve) and the Great Attractor (dotted line) as seen with the bar detector AURIGA. With a sensitivity of $10^{-23}$ Virgo will be detectable between $t=0 \mathrm{~h}$ and $t=18 \mathrm{~h}$. b) Ibidem for scalar waves. Virgo could be detectable at two main positions $t=9 \mathrm{~h}$ and $t=21 \mathrm{~h}$. The Great Attractor could be barely detected with a sensitivity of $10^{-23}$ at $t=12 \mathrm{~h}$

predicted for Virgo alone as seen from Fig. 7a. This confirms that the Virgo cluster will be the dominant source of GW events when the sensitivity will be $h_{\text {lim }}=10^{-23}$.

From Fig. 11b (AURIGA bar detector) one can see clearly that tensor waves and scalar waves have opposite phases as far as the sidereal time is concerned. The same effect is also visible from Fig. $12 \mathrm{a}$ and $\mathrm{b}$ with the NAUTILUS bar detector. Further, we see that changing the orientation of the bar by 90 deg also produces a change of sidereal time phase; The maximum in Fig. 12b for, say, tensor waves (solid curve), corresponds to a minimum in Fig. 12a. 

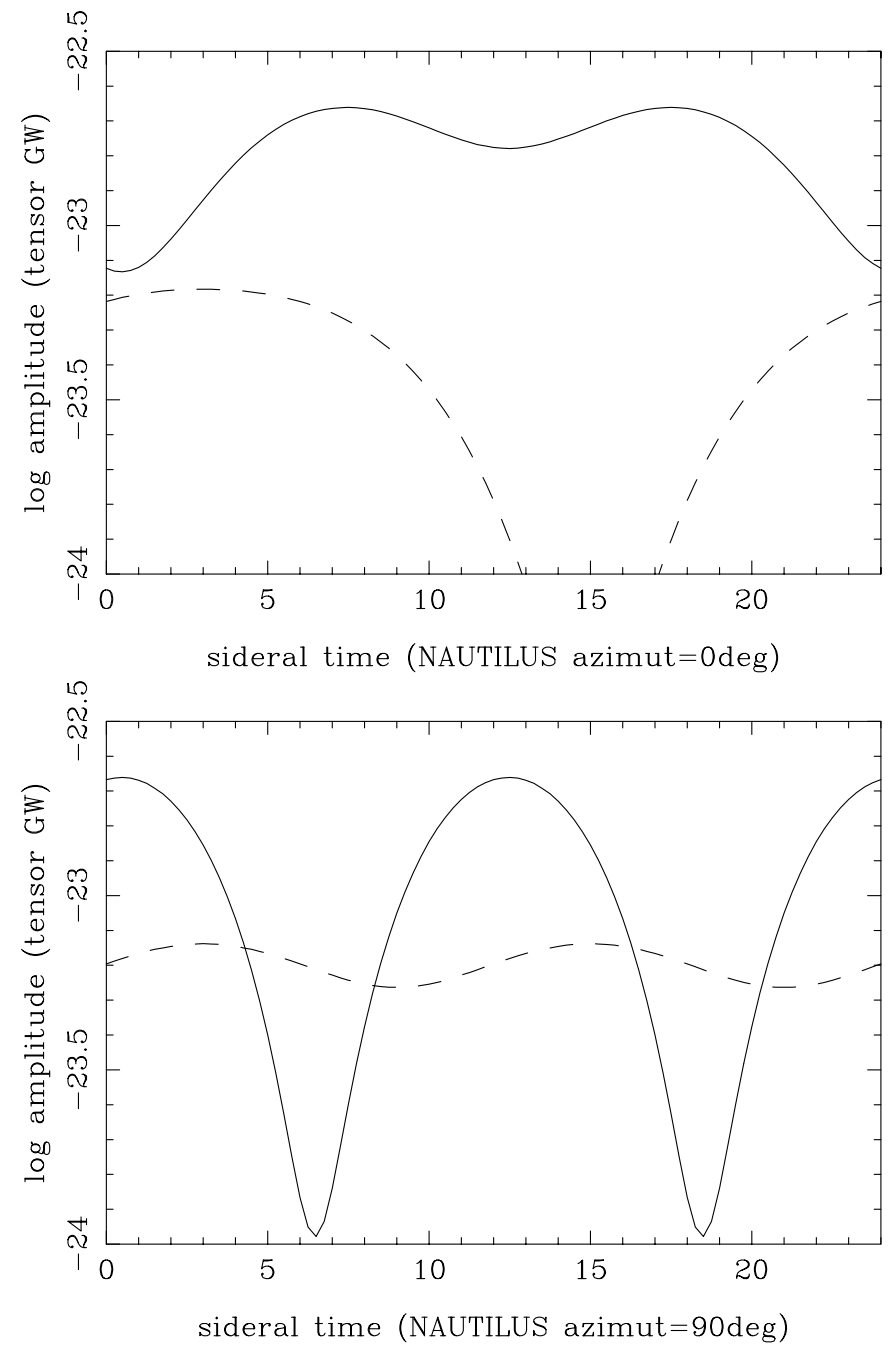

Fig. 9. a) Amplitude as a function of sidereal time for tensor GW emitted by Virgo (solid curve) and the Great Attractor (dashed curve) as seen with the bar detector NAUTILUS oriented in the direction of the north (azimuth $=0 \mathrm{deg}$ ). This figure is the same as Fig. 8a but with a shift in sidereal time. b) Ibidem with NAUTILUS oriented in the east-west direction (azimuth $=90 \mathrm{deg}$ ). Virgo could be detectable at two main positions $t=1 \mathrm{~h}$ and $t=12 \mathrm{~h}$. Figures a and $\mathrm{b}$ have opposite phases

\section{Discussion and conclusions}

From the detection of GW events with bar and interferometric detectors, one can see that, due to the different geometrical factors and to the anisotropy of the distribution of the GW sources, it is in principle possible to make the distinction between transversal tensor GW, transversal scalar GW and longitudinal scalar GW.

To demonstrate this difference we calculated the expected amplitudes and the number of events as a function of sidereal time for real positions of existing GW detectors and for real 3-dimensional distribution of galaxies within $100 \mathrm{Mpc}$. If one adopts the value for the energy of GW pulse $E_{\mathrm{gw}}=10^{-6} M_{\odot} c^{2}$ (lines $b$ in Fig. 3), then, GW events produced at the distance of the Virgo
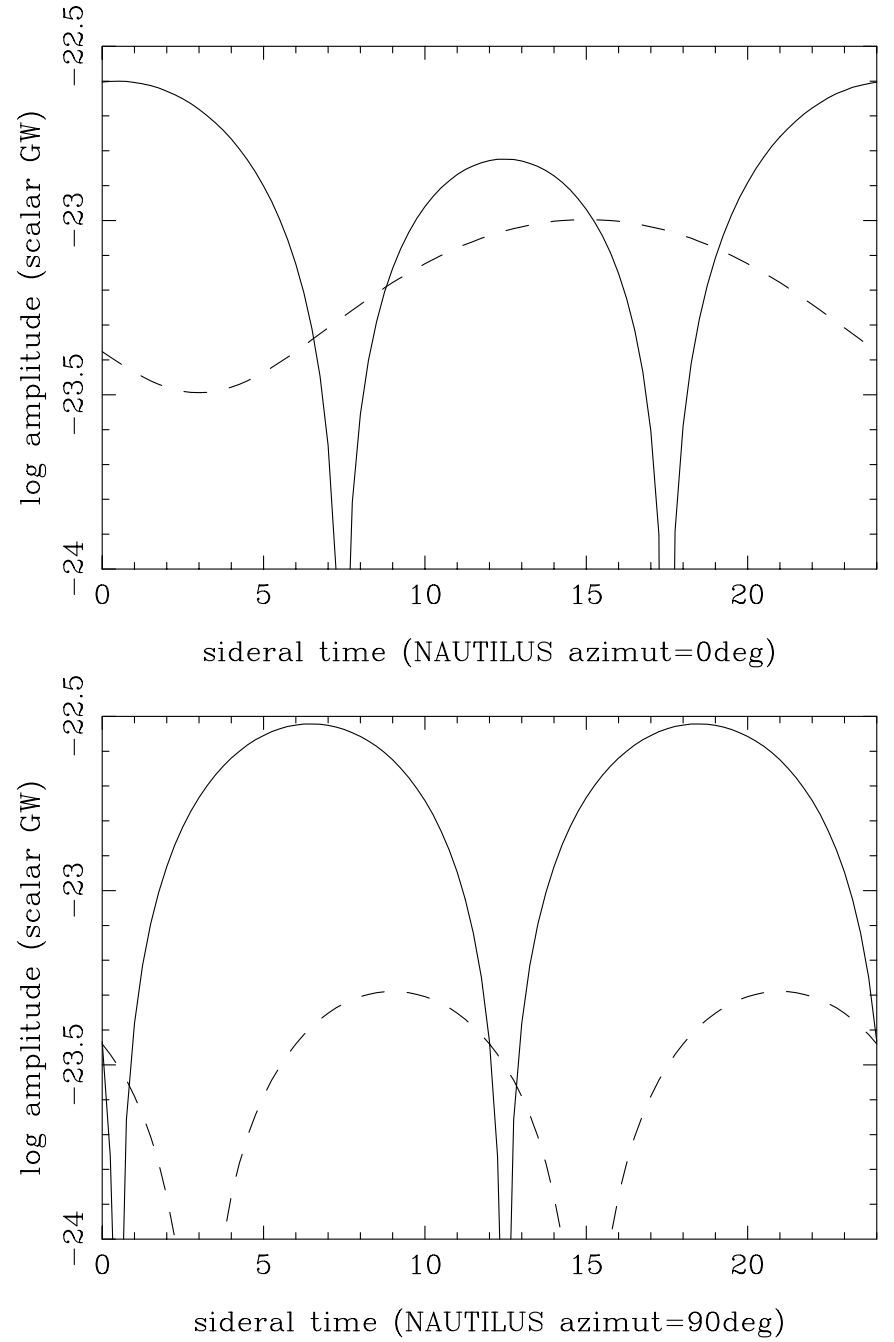

Fig. 10. a) Amplitude as a function of sidereal time for scalar GW emitted by Virgo (solid curve) and the Great Attractor (dashed curve) as seen with the bar detector NAUTILUS oriented in the direction of the north (azimuth $=0 \mathrm{deg}$ ). This figure is the same as Fig. 9a but with a shift in sidereal time. b) Ibidem with NAUTILUS oriented in the east-west direction (azimuth $=90 \mathrm{deg}$ ). Virgo could be detectable at two main positions $t=7 \mathrm{~h}$ and $t=18 \mathrm{~h}$. Again, Figs. a and b have opposite phases

cluster can be detected only with a sensitivity, yet unreachable, of $h_{\lim }=10^{-22.5}$. However if the GW energy is about $E_{\mathrm{gw}}=10^{-3} M_{\odot} c^{2}$ (case $a$ in Fig. 3) as predicted by nonaxisymmetric scenarios of $\mathrm{SN}$ core collapse, then Virgo cluster and Great Attractor would be visible with $h_{\lim }=10^{-22}$.

If the GW energy emission has value $E_{\mathrm{gw}}=$ $10^{-9} M_{\odot} c^{2}$ or less, as predicted by an axisymmetric scenario of SN explosion, then Virgo would simply be not detectable with present and forthcoming detectors. Thus, one cannot expect a high detection rate, but only serendipitous detections from very nearby SN's.

We would like to emphasize a point which seems important to us. Today there are several scenarios of GW radiation from SN core collapse even in the frame of 

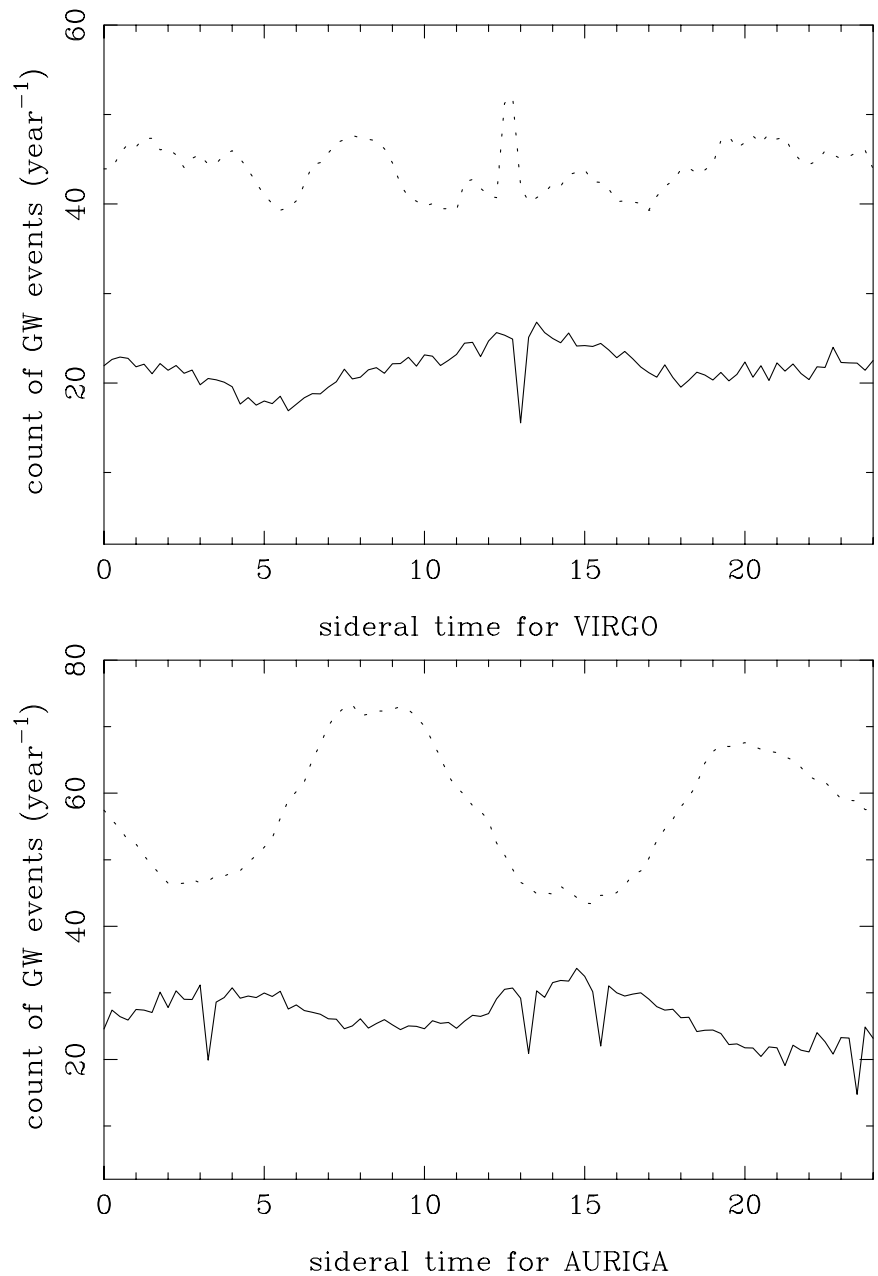

Fig. 11. a) Density of probability of GW events (number per year) for the VIRGO interferometric detector as a function of the sidereal time of the site. The calculation is made from the actual sample of galaxies described in Sect. 2. Tensor waves are shown with a solid curve, while scalar waves are given as dashed curves. These predicted counts are obtained from Eq. (6) using case b of Fig. 3 for the calculation of the observed amplitude. The sensitivity is assumed to be $h_{\lim }=10^{-23}$. Because one retrieves the expected distribution found for Virgo alone (solid curves of Fig. 7), one concludes that the Virgo cluster will be the dominant source of GW events with such a sensitivity. b) Ibidem for the AURIGA bar detector. The comparison with predicted amplitude for Virgo cluster alone also confirms that it will be the dominant source of GW events at the considered sensitivity. It is clearly visible that tensor and scalar waves have opposite phases

the General Relativity, which predicts only tensor gravitational radiation (see review by Thorne 1997). Within different scenarios, the SN core collapse may lead to a large range of radiated GW energy from $10^{-11} M_{\odot} c^{2}$ up to $10^{-2} M_{\odot} c^{2}$ and to very different forms of GW bursts, i.e. to the different spectral energy distributions and durations of GW pulses from msec up to sec and even minutes (Lai \& Shapiro 1995). It is important to recall that other relativistic and quantum gravity theories (such as string theory, the Jordan-Fierz-Brans-Dicke theory and tensor
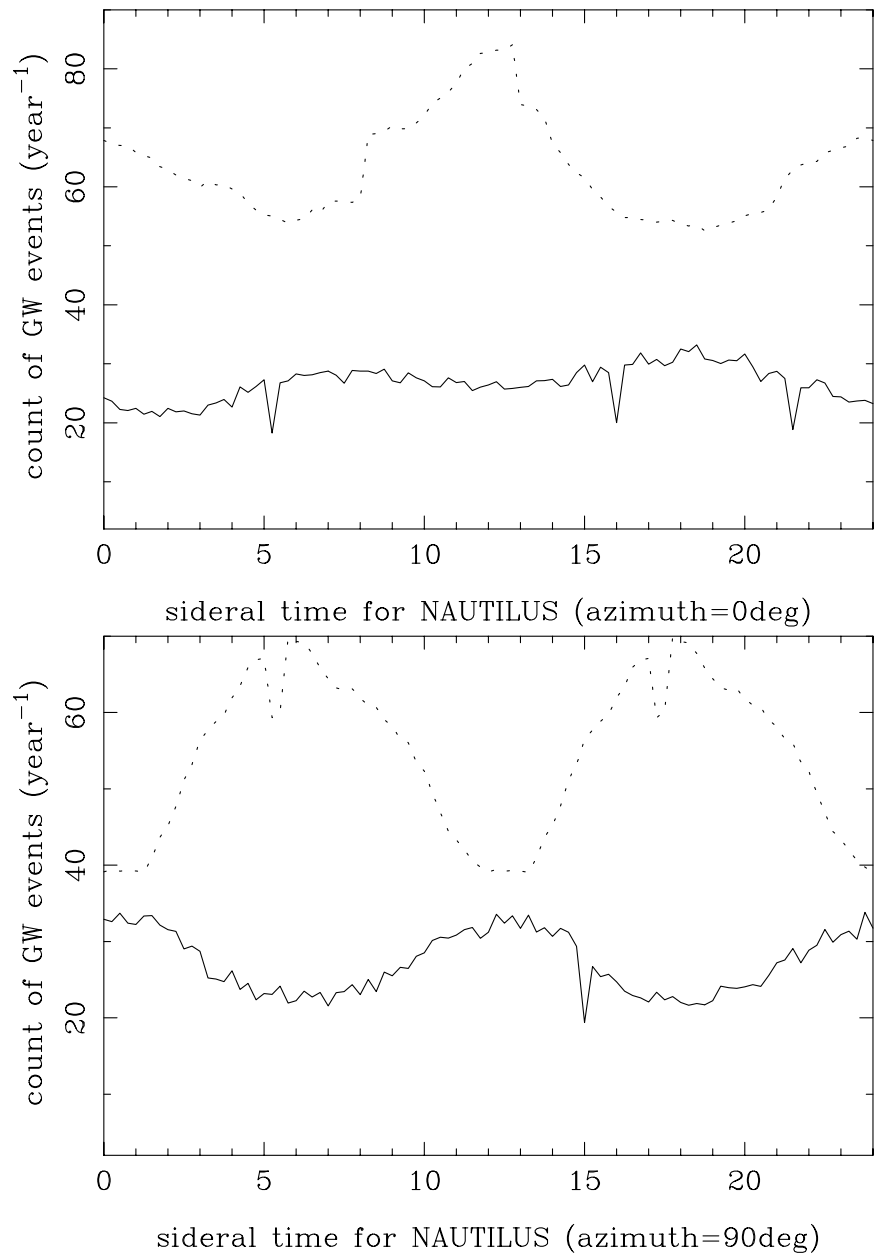

Fig. 12. a) Same as the previous figure for the NAUTILUS pointing towards the north $\left(\Phi_{\mathrm{o}}=0 \mathrm{deg}\right)$. The results and the conclusions are very similar to those obtained from Fig. 11b. Nevertheless we note that this orientation is less favorable for scalar waves in comparison with the AURIGA bar detector. b) Ibidem for the NAUTILUS pointing towards the west $\left(\Phi_{\mathrm{o}}=\right.$ $90 \mathrm{deg})$. We note the improvement of detected GW events when comparing with the previous orientation

field theory) predict scalar gravitational radiation which is generated also in the case of a spherical gravitational collapse and which may carry a large GW energy.

We would like to emphasize the importance of considering a wide range of GW burst parameters for SN core collapses by discussing the SN1987A and SN1993J events. Analysis of data recorded by Geograv for SN1987A (Amaldi et al. 1987) and by Explorer-Allegro for SN1993J (Mauceli et al. 1997), showed that there are GW candidate events, which the authors themselves do not consider as real signals because the GW energy calculated for a standard pulse with a duration $\tau_{\mathrm{g}}=1 \mathrm{~ms}$ (and hence bandwidth $1 \mathrm{kHz}$ ) gives $E_{\mathrm{gw}} \approx 10^{3} M_{\odot} c^{2}$ for both supernovae. However, in the case of pulse duration of about $1 \mathrm{~s}$ (and hence bandwidth $1 \mathrm{~Hz}$ ) the GW energy needed for producing the same GW amplitude is about $1 M_{\odot} c^{2}$. In this case the observed GW amplitudes correspond to the lines 
"a" in Fig. 3 and fit well the decrease expected from the relative distances of the two host galaxies (LMC, M 81) ${ }^{4}$.

This means that GW data analysis should be done for the interval of possible signal durations from $\mathrm{ms}$ to sec timescales. For the GW pulses longer than $1 \mathrm{~s}$ the frequency bandwidth is less than $1 \mathrm{~Hz}$ and the sensitivity of bar detectors may be essencially improved if one uses as a signal the difference between two signals coming from two resonances of a bar detector.

Let us conclude with the most secure results presented in this paper:

- We wrote a computer code to calculate the amplitude of transversal or longitudinal gravitational waves from any real source which could be detected with bar or interferometer detectors. This code allows us to fix all experimental conditions: latitude and sideral time of the detector, type and orientation of the detector, right ascension and declination of the emitting source, characteristics of the gravitational wave;

- This code has been used to calculate how the amplitude changes with sideral time of the site for existing detectors. In particular, using a point source we study the effect of the polarization of the wave in the case of tensor waves;

- Then, we compared the distribution of amplitudes along the sideral time for bar and interferometric detectors and for transversal and longitudinal gravitational waves for two dominant point sources. We found an interesting result for bar detectors (NAUTILUS) which can have different orientations. This may be used for the selection of real GW signals from the noise. The result is that it would be theoretically possible to discriminate both kinds of waves because, for a considered point source, the maxima of the distributions do not appear at the same sideral time;

- Finally, we applied the code to calculate the expected count of events generated by the actual distribution of galaxies using the gravitational energy release predicted by existing scenarios of Supernova core collapse. The result is that, with the sensitivity of GW detectors $h=10^{-22}$ and for the released GW energy $E_{\mathrm{gw}}=10^{-3}$, predicted by nonaxisymmetric scenarios of SN core collapse supernova, it is possible to detect GW events from the distance of the Virgo cluster and Great Attractor and to use the statistics of the events as a test of gravity theories.

\section{Appendix A: The longitudinal character of the scalar wave in the field gravity theory}

Feynman's field gravity theory (Feynman et al. 1995) is based on the Lagrangian formalism of the

\footnotetext{
4 The high value of energy release in a gravitational collapse could also be supported by the existence of gamma-ray bursts if they are related to SN explosions because they may generate in the electromagnetic channel an energy of about $1 M_{\odot} c^{2}$ with timescale a few seconds (Kulkarni et al. 1999; Paczynski 1999).
}

relativistic quantum field theory and presents a nongeometrical description of gravitational interaction. According to Feynman, the gravity force between two masses is caused by the exchange of gravitons which are mediators of the gravitational interaction and actually represent the quantum of the relativistic tensor field $\psi^{i k}$ in Minkowski space $\eta^{i k}$.

It is important that the problem of the physical interaction of a gravitational wave with an detector may be completely analyzed in terms of the weak field approximation where classical relativistic field theory is applicable (see Landau \& Lifshitz 1971).

First, let us consider the general problem of the motion of a relativistic test particle having rest-mass $m_{0}$, 4 -velocity $u^{i}$, and 3 -velocity $\boldsymbol{v}$ in the gravitational field described by the symmetric tensor potential $\psi^{i k}$ in the flat Minkowski space-time. The Cartesian coordinates always exist and the metric tensor is $\eta^{i k}=\operatorname{diag}(1,-1,-1,-1)$ (we utilize notations of the text-book Landau \& Lifshitz 1971).

To derive the equation of motion for test particles in the frame of the field gravity theory we start from the stationary action principle in the form

$\delta S=\delta\left(\frac{1}{c} \int\left(\Lambda_{(\mathrm{p})}+\Lambda_{(\mathrm{int})}\right) \mathrm{d} \Omega\right)=0$

where the variation of the action is made with respect to the particle trajectories $\delta x^{i}$ for fixed gravitational potential $\psi^{i k}(t, \boldsymbol{r})$, and $\mathrm{d} \Omega$ is the element of the 4 -volume.

The free particle Lagrangian is

$\Lambda_{(\mathrm{p})}=-\eta_{i k} T_{(\mathrm{p})}^{i k}$

and the interaction Lagrangian is

$\Lambda_{(\mathrm{int})}=-\frac{1}{c^{2}} \psi_{i k} T_{(\mathrm{p})}^{i k}$

where the energy-momentum tensor of the test point particle is

$T_{(\mathrm{p})}^{i k}=m_{0} c^{2} \delta\left(\boldsymbol{r}-\boldsymbol{r}_{\boldsymbol{p}}\right)\left\{1-\frac{v^{2}}{c^{2}}\right\}^{1 / 2} u^{i} u^{k}$.

The result of variation gives the equations of motion in the form (Kalman 1961; Baryshev 1986):

$A_{k}^{i} \frac{\mathrm{d} p^{k}}{\mathrm{~d} s}=-m_{0} B_{k l}^{i} u^{k} u^{l}$

where $p^{k}=m_{0} c u^{k}$ is 4 -momentum of the test particle, $(\cdot)_{, i}=\frac{\partial(\cdot)}{\partial x^{i}}$ and

$A_{k}^{i}=\left(1-\frac{1}{c^{2}} \psi_{n l} u^{n} u^{l}\right) \eta_{k}^{i}-\frac{2}{c^{2}} \psi_{k n} u^{n} u^{i}+\frac{2}{c^{2}} \psi_{k}^{i}$

$B_{k l}^{i}=\frac{2}{c^{2}} \psi_{k, l}^{i}-\frac{1}{c^{2}} \psi_{k l}{ }^{i}-\frac{1}{c^{2}} \psi_{k l, n} u^{n} u^{i}$.

In Eq. (A.5) the rest mass of the test particle may be canceled, hence in the field gravity theory $m_{\mathrm{in}}=m_{\mathrm{g}}=m_{0}$ without the initial equivalence postulate (Thirring 1961). 
In agreement with Eq. (A.5) all classical relativistic postNewtonian gravity effects have the same values as in general relativity (see e.g. Baryshev 1995).

The scalar gravitational wave in the field gravity theory is generated by the trace of the energy momentum tensor $T=\eta_{i k} T^{i k}$ of the sources of the gravitational potential (Baryshev 1982, 1995; Sokolov 1992) and may be expressed in the form:

$\psi^{i k}(t, \boldsymbol{x})=\frac{1}{4} \psi(t, \boldsymbol{x}) \eta^{i k}$

where $\psi=\eta_{i k} \psi^{i k}$ is the trace of the tensor potential $\psi^{i k}$ and hence is a 4-scalar field in Minkowski space.

Substituting Eq. (A.8) in Eq. (A.5) and taking into account the weak field approximation we get the following equation of motion of the test particle in scalar gravitational potential:

$\frac{\mathrm{d} u^{i}}{\mathrm{~d} s}=\frac{1}{4 c^{2}} \psi^{, i}-\frac{1}{4 c^{2}} \psi_{, l} u^{l} u^{i}$.

Spatial components of this equation $(i=\alpha)$ give the expression for the gravity force acting on the test particle:

$$
\begin{aligned}
\frac{\mathrm{d} \boldsymbol{p}}{\mathrm{d} t}= & -\frac{m_{0}}{4 \sqrt{1-\frac{v^{2}}{c^{2}}}}\left[\left(1-\frac{v^{2}}{c^{2}}\right) \nabla \psi\right. \\
& \left.+\frac{\boldsymbol{v}}{c}\left(\frac{\partial \psi}{c \partial t}+\frac{\boldsymbol{v}}{c} \cdot \nabla \psi\right)\right] .
\end{aligned}
$$

The corresponding 3-acceleration of the test particle is

$$
\frac{\mathrm{d} \boldsymbol{v}}{\mathrm{d} t}=-\frac{1-\frac{v^{2}}{c^{2}}}{4}\left(\nabla \psi+\frac{\boldsymbol{v}}{c} \frac{\partial \psi}{c \partial t}\right) .
$$

The time component $(i=0)$ of the Eq. (A.9) gives the work produced by the scalar gravitational wave:

$\frac{\mathrm{d} E_{k}}{\mathrm{~d} t}=-\frac{m_{0}}{4 \sqrt{1-\frac{v^{2}}{c^{2}}}}\left(\boldsymbol{v} \cdot \nabla \psi+\frac{v^{2}}{c^{2}} \frac{\partial \psi}{c \partial t}\right)$

where $E_{k}$ is the kinetic energy of the particle. For the case of slow motion of the test particle $(v / c \ll 1)$ we get for the gravity force:

$\frac{\mathrm{d} \boldsymbol{p}}{\mathrm{d} t}=-\frac{m_{0}}{4} \nabla \psi$

for 3-acceleration of the test particle:

$\frac{\mathrm{d} \boldsymbol{v}}{\mathrm{d} t}=-\frac{1}{4} \nabla \psi$

and for the work of the gravity force:

$\frac{\mathrm{d} E_{k}}{\mathrm{~d} t}=-\frac{m_{0}}{4}(\boldsymbol{v} \cdot \nabla \psi)$.

The scalar plane monochromatic GW in the system of coordinates with the $z$-axis directed along the wave propagation may be written in the form:

$\psi_{i k}=\frac{1}{4} \psi(t, z) \eta_{i k}$ $\psi_{i k}=h_{0} \cos (\omega t-k z) \operatorname{diag}(1,-1,-1,-1)$

where $h_{0} \ll c^{2}$ is the amplitude of the wave, $\omega=2 \pi \nu$, $k=\omega / c=2 \pi / \lambda$.

For the scalar gravitational potential Eq. (A.17) the 3-acceleration Eq. (A.14) of the test particle is

$\frac{\mathrm{d} v_{x}}{\mathrm{~d} t}=\frac{\mathrm{d} v_{y}}{\mathrm{~d} t}=0$

$\frac{\mathrm{d} v_{z}}{\mathrm{~d} t}=\frac{1}{4 c} \frac{\partial \psi}{\partial t}$.

According to Eq. (A.19) under the action of the scalar gravitational wave a test mass undergo small longitudinal oscillations in the direction of wave propagation ( $z$-axis):

$z(t)=\frac{\lambda}{2 \pi} \frac{h_{0}}{c^{2}} \sin \left(\omega t-k z_{0}\right)+z_{0}$.

The relative distance between two particles $\Delta z(t)=$ $z_{1}(t)-z_{2}(t)=l(t)$ gives the relative oscillations $\Delta l(t)=$ $l(t)-l_{0}=\Delta l_{0} \cos (\omega t+\alpha)$, which may be detected by gravitational detector.

The very important property of the scalar gravitational potential is that it does not interact with the electromagnetic field. Indeed, the interaction Lagrangian for the potential Eq. (A.8) is

$\Lambda_{(\mathrm{int})}=-\frac{1}{c^{2}} \psi_{i k} T_{(\mathrm{em})}^{i k}=0$

because the trace of the energy momentum tensor of the electromagnetic field $T_{(\mathrm{em})}=0$. This means that the above analysis is sufficient for the study of the response of the bar or interferometric detectors where the distances between test particles are measured by means of the electromagnetic field.

For a detector with the length $l_{0} \ll \lambda$ the amplitude of the relative displacement $\Delta l_{0} / l_{0}$ between two test particles, which is measured by the detector is

$\frac{\Delta l_{0}}{l_{0}}=h_{0} \cos \theta$

where $h_{0}$ is the amplitude of the scalar wave Eq. (A.17), and $\theta$ is the angle between the direction of the wave propagation and the line connecting the two masses.

For a bar detector the integral cross section for the scalar gravitational wave having $\Delta \nu_{\mathrm{g}}>\Delta \nu_{\mathrm{a}}$ is given by (Baryshev 1997):

$\sigma_{(\text {scalar })}=\frac{8}{\pi} \frac{G}{c^{3}} M V_{\mathrm{s}}^{2} \cos ^{2} \theta$

where $M$ is the mass of the cylinder, $V_{\mathrm{s}}=\omega_{0} L / \pi$ is the speed of sound in the cylinder, $\omega_{0}$ is resonance angular frequency of the bar, $L$ is the length of the cylinder and $\theta$ is the angle between the bar axis and the direction of the wave propagation. The factor $\cos \theta$ in Eqs. (A.22, A.23) shows the longitudinal character of the scalar gravitational wave. 
Acknowledgements. We thank Giovanni Pallottino and Paolo Bonifazi for giving parameters of bar detectors and comments, Pekka Teerikorpi and Vladimir Sokolov for useful discussions which helped to improve the text. Y.B. thanks the President of the University of Lyon I for inviting him on a temporary position. We thank the anonymous referee for usefull coments.

\section{References}

Amaldi, E., Bonifazi, P., Castellano, M. G., et al. 1987, Europhys. Lett., 3, 1325

Amaldi, E., \& Pizzella, G. 1979, in Relativity, Quanta and Cosmology in the development of the scientific thought of Albert Einstein (Johnson Rep. Corp., Acad. Press), 241

Baryshev, Yu. V. 1982, Astrophys., 18, 58

Baryshev, Yu. V. 1986, Vestnik Leningrad State Univ., Ser. 1, No. 4, 113

Baryshev, Yu. V. 1995, in the Proceedings of the First Edoardo Amaldi Conference on Gravitational Wave Experiment, ed. E. Coccia, G. Pizzella, \& F. Ronga (World Scientific), 251

Baryshev, Yu. V. 1997, Astrophys., 40, 244

Baryshev, Yu. V., \& Sokolov, V. V. 1984, Astrophys., 21, 548

Bianchi, M., Brunetti, M., Coccia, E., Fucito, F., \& Lobo, J. A. 1998, Phys. Rev. D, 57, 4525

Bonnazzola, S., \& Marck, J. A. 1993, A\&A, 267, 623

Bonnell, I. A., \& Pringle, J. E. 1995, MNRAS, 273,L12

Brunetti, M., Coccia, E., Fafone, V., \& Fucito, F. 1999, Phys. Rev. D, 59, 044027

Burrows, A. 2000, Nature, 403, 727

Damour, T. 1999 [gr-qc/9904057]

Damour, T., \& Esposito-Farese, G. 1992, Class. Quantum Grav, 9, 2093

Damour, T., \& Esposito-Farese, G. 1996, Phys. Rev. D, 54, 1474

Damour, T., \& Esposito-Farese, G. 1998, Phys. Rev. D, 58, 042001

Davis, M. 1997, in the Proceedings of the Princeton Conference, Critical Dialogues in Cosmology, ed. N. Turok (World Scientific)

Davis, M., \& Peebles, P. J. E. 1983, ApJ, 267, 465

Dressler, A., Faber, S. M., Burstein, D., et al. 1987, ApJ, 313, L37

Ferrari, V., Matarrese, S., \& Schneider, R. 1999, MNRAS, 303, 247

Feynman, R., Morinigo, F., \& Wagner, W. 1995, Feynman Lectures on Gravitation (Addison-Wesley Publ.Co.)

Gasperini, M. 1999 [gr-qc/9910019]

Harada, T., Chiba, T., Nakao, K., \& Nakamura, T. 1997, Phys. Rev. D, 55, 2024

Kalman, G. 1961, Phys. Rev., 123, 384

Kalogera, V. 1999 [astro-ph/9911110]
Khokhlov, A. M., Yi, I., Hoflich, P. A., et al. 1999, ApJ, 524, L107

Kraan-Korteweg, R. 2000, A\&AS, 141, 123

Kulkarni, S. R., Djorgovski, S. G., Odewahn, S. C., et al. 1999, Nature, 398, 389

Lai, D., Shapiro, S. T. 1995, ApJ, 442, 259

Landau, L. D., \& Lifshitz, E. M. 1971, The Classical Theory of Field (Pergamon Press, N.Y.)

Lipunov, V. M., Nazin, S. N., Panchenko, I. E., Postnov, K. A., \& Prokhorov, M. E. 1995, A\&A, 298, 677

Lipunov, V. M., Postnov, K. A., \& Prokhorov, M. E. 1997, New Astr., 2, 43

MacFadyen, A., \& Woosley, S. E. 1999, ApJ, 524, 262

Maggiore, M., \& Nicolis, A. 2000 [gr-qc/9907055]

Mauceli, E., Geng, Z. K., Hamilton, W. O., et al. 1997, Phys. Rev. D, 56, 6081

Nakao, K., Harada, T., Shibata, M., Kawamura, S., \& Nakamura, T. 2000 [gr-qc/0006079]

Novak, J., Ibanez, J. 1999 [gr-qc/9911298]

Paturel, G., Bottinelli, L., Gouguenheim, L., \& Fouqué, P. 1987, A\&AS, 189, 1

Paczynski, B. 1999 [astro-ph/9909048]

Pietronero, L., Montuori, M., \& Sylos Labini, F. 1997, in the Proceedings of the Princeton Conference Critical Dialogues in Cosmology, ed. N. Turok (World Scientific)

Pizzella, G. 1989, in Gravitational Wave Data Analysis ed. by B. F. Schutz (Kluwer Acad. Publishers), 173

Portegies, Zwart, S., \& McMillan, S. 1999 [astro-ph/9910061]

Prodi, G., Heng, I. S., Allen, Z. A., et al. 2000, in Proc. of the GWDAW99, in press

Schutz, B. F., \& Tinto, M. 1987, MN, 224, 131

Shibata, M., Nakao, K., \& Nakamura, T. 1994, Phys. Rev. D, 50,7304

Sokolov, V. V. 1992, Ap. Sp. Sci. 198, 53

Stark, R. F., \& Piran, T. 1985, Phys. Rev. Lett., 55, 891

Straumann, N. 2000 [astro-ph/0006423]

Sylos Labini, F., Montuori, M., \& Pietronero, L. 1998, Phys. Rep., 293, 61

Teerikorpi, P., Hanski, M., Theureau, G., et al. 1998, A\&A, 334,395

Thirring, W. E. 1961, Ann. Phys., 16, 96

Thorn, K. S. 1987, in Three Hundred Years of Gravitation (Cambridge University Press), 330

Thorn, K. S. 1997 [gr-qc/9706079]

van den Bergh, S., \& Tammann, G. 1991, ARA\&A, 29, 363

Wang, L., Howell, D. A., Hoflich, P., \& Wheeler, J. C. 1999 [astro-ph/9912033]

Wheeler, J. C., Yi, I., Hoflich, P., \& Wang, L. 2000 [astro-ph/9909293]

Zwerger, T., \& Muller, E. 1997, A\&A, 320, 209 